\title{
Ligand-Field Theory-Based Analysis of the Adsorption Properties of Ruthenium Nanoparticles
}

\author{
del Rosal, Iker ${ }^{\dagger}$; Mercy, Maxime ${ }^{\dagger, \ddagger} ;$ Gerber, lann C. ${ }^{\dagger}$ Poteau, Romuald ${ }^{\dagger *}$
}

September 16, 2013

\begin{abstract}
†Université de Toulouse; INSA, UPS, CNRS; LPCNO (IRSAMC), 135 avenue de Rangueil, F-31077
Toulouse, France

$\ddagger$ Present address: Department of Chemistry, University College London, 20 Gordon Street, London WC1H $O A J$, United Kingdom

idel_ros@insa-toulouse.fr, m.mercy@ucl.ac.uk, iann.gerber@insa-toulouse.fr, *romuald.poteau@univ-tlse3.fr
\end{abstract}

\begin{abstract}
The experimental design of improved nanocatalysts is usually based on shape control and is surfaceligand dependent. First-principle calculations can guide their design, both in terms of activity and selectivity, provided that theoretical descriptors can be defined and used in a prescreening process. As a consequence of the Sabatier principle and of the Brønsted-Evans-Polanyi relationship, an important prerequisite before optimizing the catalytic properties of nanoparticles is the knowledge of the selective adsorption strengths of reactants at their surface. We report here adsorption energies of $\mathrm{X}\left(\mathrm{H}, \mathrm{CH}_{3}\right)$ and $\mathrm{L}\left(\mathrm{PH}_{3}, \mathrm{CO}\right)$ ligands at the surface of bare ruthenium nanoclusters $\mathrm{Ru}_{n}(n=55$ and 147) calculated at the DFT level. Their dependence on the topology of the adsorption sites as well as on the size and shape of the nanoparticles (NPs) is rationalized with local descriptors derived from the so-called $d$-band center model. Defining the descriptors involves the determination of the energy of effective $d$ atomic orbitals for each surface atom. Such a ligand field theory-like model is in close relation with frontier molecular orbital theory, a cornerstone of rational chemical synthesis. The descriptors are depicted as color maps which straightforwardly yield possible reactivity spots. The adsorption map of a large spherical hcp cluster $\left(\mathrm{Ru}_{288}\right)$ nicely confirms the remarkable activity of steps, the so-called $\mathrm{B}_{5}$ sites. The predictive character of this conceptual DFT approach should apply to other transition metal NPs and it could be a useful guide to the design of efficient nanocatalysts bearing sites with a specific activity.
\end{abstract}

Keywords. DFT ; Ruthenium ; Nanocatalysis ; Sabatier Principle ; Reactivity Descriptors

Nanoparticles (NPs) exhibit physical and chemical properties intermediate between those of small molecular compounds and the bulk material, resulting from surface or quantum size effects. ${ }^{1}$ Special sites ${ }^{2}$ are also known to play a role in heterogeneous catalysis ${ }^{3}$ and in synthesis over nanoparticles. ${ }^{4,5}$ More efficient nanocatalysts need to be developed, yet the task of discovering novel alternatives has proven to be extremely challenging. We focus in the present paper on ruthenium NPs (RuNPs), revisiting some key concepts by means of first-principles DFT calculations. Ruthenium and its derivatives constitute an important class of catalysts, involved in hydrogenolysis reactions, ${ }^{6}$ olefin metathesis, ${ }^{7}$ hydrogenation of aromatic compounds, ${ }^{8,9}$ and the Fischer-Tropsch reaction. ${ }^{10,11} \mathrm{It}$ is a catalyst of utmost importance which deserves a deeper understanding of its surface properties under the NP state, a range of sizes with possible quantum 
size effects. The surface properties being ruled by the intrinsic electronic structure of the metal NPs, quantum chemistry is expected to provide valuable insights and to guide the development of new catalysts. But, is theoretical chemistry able to provide clues about the structure and reactivity of colloidal nanoparticles (NPs) as well as useful guidelines to experimentalists, regarding the complexity of such problems? Despite some recent spectacular results (see ref 12 and references therein), quantum chemistry is not mature enough to reach a routine understanding of the activity of nanoparticles similar to that gained on organometallic complexes in homogeneous catalysis ${ }^{13}$ and to contribute to the rational design of nanocatalysts. State-ofthe-art quantum chemistry studies of homogeneous and heterogeneous ${ }^{14,15}$ processes involve the exploration of potential energy surfaces in order to elucidate multi-step reaction paths. But, complex reactions that occur at the surface of NPs have scarcely been investigated with such computational approaches. ${ }^{16,17}$ The reason lies in the complexity and the diversity of the metal sites, the presence of ancillary ligands and other surface species, which make such calculations difficult to achieve with current computational quantum chemistry tools. Theoretical chemists get around this problem by considering NPs as faceted crystals, i.e. as a juxtaposition of flat, kinked and stepped surfaces. They can thus take advantage of periodic-boundary DFT methods to use chain-of-states based methods for finding minimum energy pathways. ${ }^{18-20}$ On the other hand, it is also possible to consider metallic clusters as scale models of NPs, usually made of less than a hundred metal atoms. ${ }^{21-31}$ Both approaches share the same drawback: they do not consider the essence of NPs, i.e. the fundamental question of quantum-size effects. The outrageous cost of the quantum chemistry exploration of reaction pathways as well as such finite-size effect challenges the in silico understanding and design of selective and catalytically efficient NPs.

We propose to tackle this problem by developing DFT-based reactivity descriptors aiming at predicting the local activity of RuNPs. They are derived from the $d$-band center model variant of Hammer and Nørskov, ${ }^{32,33}$ widely used for explaining trends in the catalytic activity of metal surfaces. Within this model, a $d$-band center parameter $\varepsilon_{d}$, is computed as the normalized, energy-weighted integral of the density of states (DOS), projected onto all $d$ atomic orbitals of the surface atoms which characterize a given adsorption site. It is important to underline that the information contained in the DOS is reduced to a single energy level, making a connection with frontier orbital theory. Despite its simplicity, this monoelectronic descriptor has proved to be very powerful to explain trends in adsorption energies. A linear relationship between the center of the $d$-band and the binding energy of the adsorbate to the surface is often observed: the closer to the Fermi level, the stronger the adsorption energy. According to the Brønsted-Evans-Polanyi (BEP) relationship 34,35 and to the Sabatier principle of maximizing catalytic activity by optimizing the adsorption energy of reactants, ${ }^{36-39}$ this has consequences well beyond the understanding of trends in adsorption energies. Indeed, the BEP equation relates the change in activation energy of a reaction to the change in the reaction energy: $\delta E_{\text {act }}=\alpha \delta E_{r}$. As a consequence, the catalytic activity of heterogeneous catalysts within the periodic table is well described by Balandin's volcano relations between reaction rates and adsorption energies. ${ }^{38,40}$ It has been extensively and successfully used in the analysis of surface elementary steps of heterogeneous catalytic processes. ${ }^{41,42}$ Note that this approach was used to guide the design of a new CoMo-based alloy catalyst for ammonia synthesis. ${ }^{43}$ If we turn to the matter of selectivity at the surface of NPs, the activity of a step or kink can also be inferred from the comparison of $\varepsilon_{d}$ calculated by selecting appropriate surface atoms in different $\{h k l\}$ crystallographic planes. ${ }^{3}$ Nonetheless, owing to the surface complexity of moderate size transition metal NPs and to the possible presence of defects and special sites, a model aiming at rationalizing adsorption properties at their surface should be able to go beyond the simple picture given by a $d$-band center average value, by taking into account the individual contributions of $d$ AOs, as it is done in molecular inorganic chemistry. Another interesting approach aiming at generating $a b$ 
initio quantitative descriptors of bond strengths in heterogeneous catalysts has also been proposed, ${ }^{37,44}$ but it does not seem to be easily transferable to NPs. In a sense, the frontier orbital equation is more powerful, since a chemical interaction can not only be analyzed from energy levels of the interacting molecules, but also from the overlap between them, which serves as basis for understanding the selectivity observed in some reactions. ${ }^{45}$ This is why we propose a new formulation of the $d$-band center model that goes beyond its original formulation by yielding a local electronic fingerprint of each surface site of a nanocatalyst.

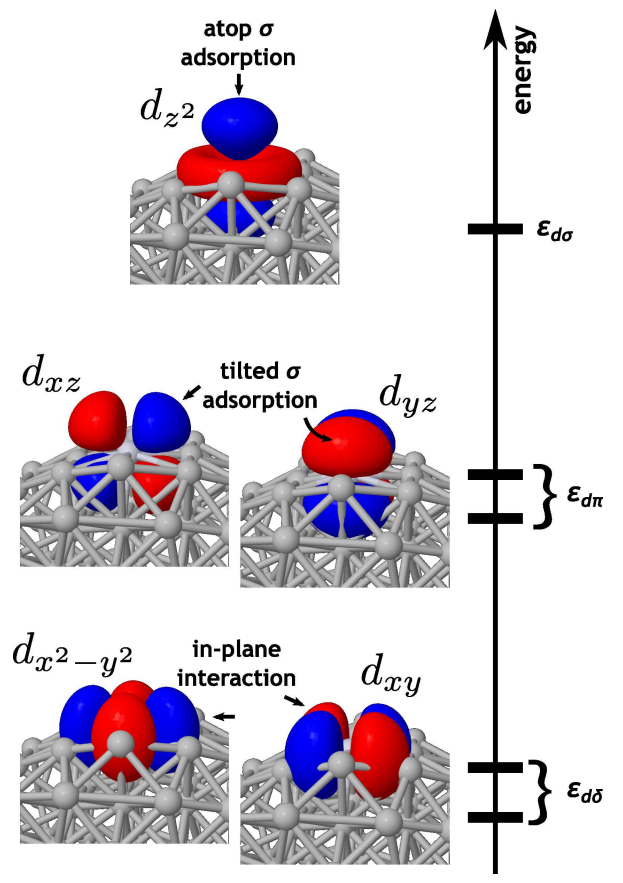

Scheme 1: effective $d$ AOs.

We first check that the $\mathrm{d}$-band center value calculated for all surface atoms of several $\mathrm{Ru}_{n}$ clusters, $\left\langle\varepsilon_{d}\right\rangle$, accounts for the simultaneous adsorption of $\mathrm{H}$ atoms. The local adsorption strength of hydrides at the surface of a $\mathrm{Ru}_{55}$ cluster is then analyzed and interpreted by an on-site $d$-band center descriptor (hereafter noted $\left.\bar{\varepsilon}_{d}\left(\mu_{k}\right)\right)$. It is depicted as a color map that gives a straightforward point of view of the expected adsorption strength at the surface of RuNPs. The predictive ability of this descriptor is then assessed by calculating the adsorption energy of $\mathrm{CO}, \mathrm{CH}_{3}$ and $\mathrm{PH}_{3}$ ligands on various sites of $\mathrm{Ru}_{n}$ clusters $(n=55$, 147). The apparent discrepancies are accounted for by introducing new descriptors that give an indication of the $\sigma, \pi$ and $\delta$ affinities of adsorption sites. Based on a meticulous analysis of these results, we formulate the powerful idea to reduce the huge amount of information contained in the electronic structure of metal NPs to effective $d$ orbitals for each metal atom of the surface (Scheme 1). Following these lines, an analysis of the electronic features of an hcp NP with realistic size $\left(\mathrm{Ru}_{288}\right)$ is provided. This analysis agrees with the well-known special site nature of steps in such ruthenium NPs. We finally conclude that our approach which combines both ligand-field theory and a related fragment molecular orbitals method may be a very useful way to identify the most active sites at the surface of a metal NP and we propose a conjecture related to the Sabatier principle.

\section{Model and results}

The original $\boldsymbol{d}$-band center model. A comprehensive model is needed to understand the DFT adsorption energies calculated in this study. It has been shown in several studies that the adsorption strength of chemical species on metal surfaces can be understood in terms of Hammer and Nørskov's $d$-band center 
model. ${ }^{32,33,46}$ It is not so different from Hoffmann's approach which is more straightforwardly related to frontier orbital theory. ${ }^{47}$ Within this model, the interaction between an adsorbate and a surface is separated into two contributions. First the adsorbate interacts with the transition metal $s p$ states and then the contribution of $d$ states is taken into account $\left(\Delta E=\Delta E_{s p}+\Delta E_{d}\right)$. According to this model, $\Delta E_{s p}$ coupling can be considered to be essentially a constant. The main contribution to the variations in bond energy from one transition metal to the next comes from the coupling to the metal $d$ states $\left(\Delta E_{d}\right)$. In a simplified description, the stabilization energy is given by a second-order perturbation theory term, $\Delta E_{d}=V^{2} /\left|\varepsilon_{\text {ads }}-\varepsilon_{d}\right|$, where $V$ is the coupling element between the adsorbate state of energy $\varepsilon_{\text {ads }}$ and the metal $d$ states centered at $\varepsilon_{d}$. The $d$-band center, $\bar{\varepsilon}_{d}\left(\mu_{k}\right)$, is then computed as the normalized, energy-weighted integral of the density of states (DOS), projected onto all $d$ atomic orbitals (AOs) of the surface atoms which characterize the $\mu_{k}$ coordination site:

$$
\bar{\varepsilon}_{d}\left(\mu_{k}\right)=\frac{\left(\sum_{\alpha \in \mu_{k}} \sum_{m} \int_{E_{\min }}^{E_{\max }} \epsilon n_{d_{m}}(\alpha, \epsilon) d \epsilon\right)}{\left(\sum_{\alpha \in \mu_{k}} \sum_{m} \int_{E_{\min }}^{E_{\max }} n_{d_{m}}(\alpha, \epsilon) d \epsilon\right)}
$$

where $m$ runs over the five $d \mathrm{AOs}$ and $n_{d}(\alpha)$ is the atom-projected density of states on the $d_{m} \mathrm{AO}$ of atom $\alpha ; \mu_{k}$ is reminiscent of the symbol which designates bridging ligands in coordination chemistry and the bar sign above $\varepsilon_{d}$ means that it is averaged over all $d$ AOs.

Although $E_{\text {min }}$ is readily set to the bottom of the occupied $d$-band, the $d$-band center cannot be defined unambiguously within a plane-wave approach. Following a previous suggestion of Stroppa and Kresse, ${ }^{48}$ we chose to calculate the center of gravity of the occupied $d$-band, i.e. we integrated up to the Fermi level $E_{F}$, which is a lower bound for the $d$-band center usually calculated (see also a short discussion in the SI).

The interaction between an adsorbate and a metal surface is based on few parameters only, including the filling, average energy and width of the localized $d$-band of the metal surface, the adsorbate molecular orbital energies and the geometric overlap between the surface and adsorbate orbitals. We shall now recall a key point of frontier orbital theory, dealing with the adsorption of organic ligands on middle transition metal surfaces. In addition to the so-called two-electron, two-orbital stabilizing interactions, the four-electron, two-orbital interactions may be attractive, owing to the partial filling of the $d$-band. It turns out that this two-orbital, four-electron interaction which is related to steric effects in orbital theory applied to molecules, may involve an additional stabilizing charge transfer from ligands to the surface. This interaction again becomes a destabilizing agent in the case of very late transition metal surfaces. The stabilizing fourelectron, two-orbital interaction being partially involved in the adsorption of species on ruthenium surfaces, setting up the integration upper bound to the Fermi level makes sense. In addition, it is expected to capture the trends in case of a metal to ligand $\pi *$ backbonding.

But in its original formulation, the $\bar{\varepsilon}_{d}$ descriptor may not be powerful and accurate enough to account for selective processes that occur at the surface of NPs, since it is an average value. Yet, orbital interactions between metal atoms and ligands is ruled by the symmetry of the individual $d$ AOs. ${ }^{49}$. This is a strong limitation of the $d$-band center model and a conceptual difference with respect to molecular orbital theory. ${ }^{50,51}$ Unfortunately, the intrinsic chemical properties at the surface of transition metal NPs do not lie within a few states around the Fermi level. This is why we propose a model that combines the powerful ligand-field theory with the reduction of the information contained in the projected DOS to one or a few effective energy levels.

Optimal coverage of metal surfaces by $\mathbf{H}$ atoms. Here, we investigate the possible relationship between the average $d$-band center of surface atoms, $\left\langle\varepsilon_{d}\right\rangle$, and the dissociative adsorption energy of hydrides, 


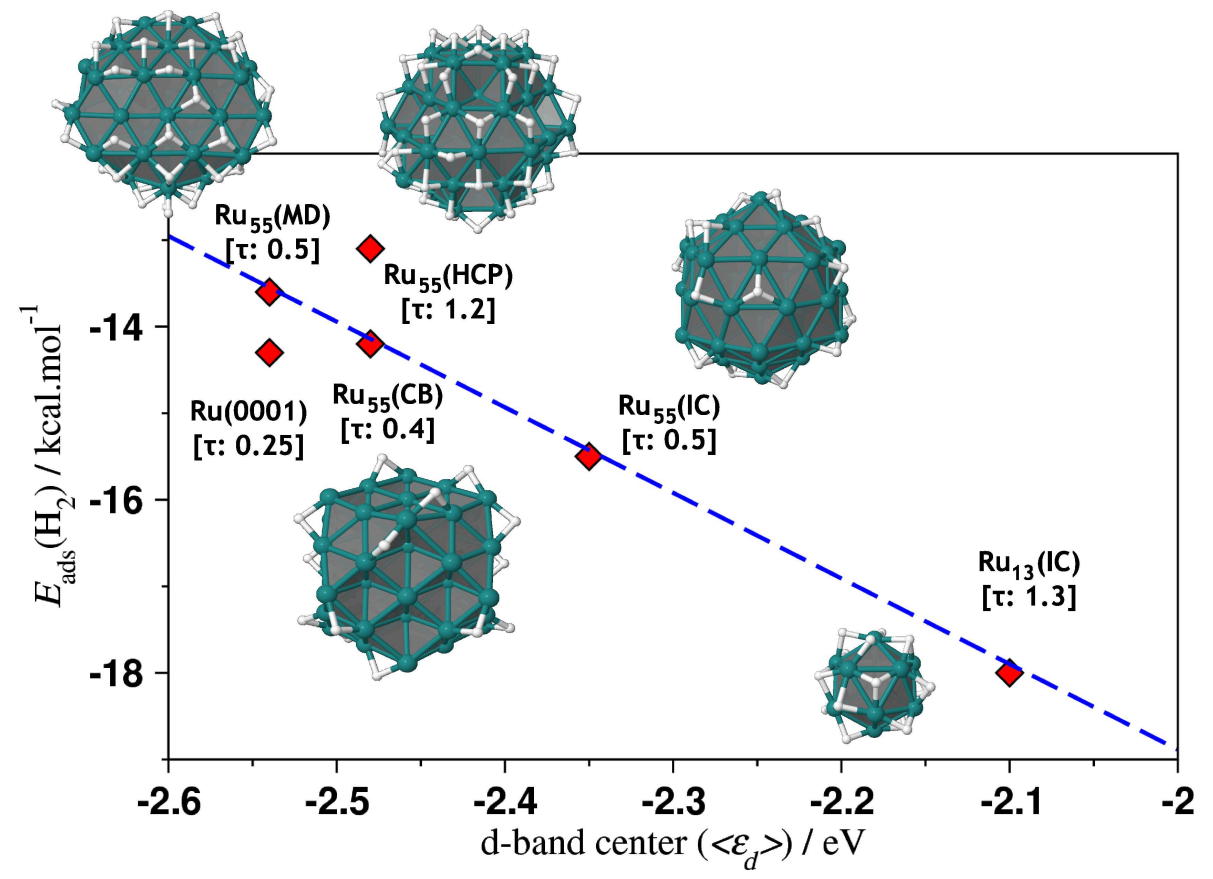

Figure 1: Plot of the average $\mathrm{H}_{2}$ dissociative adsorption energy $\left(E_{\text {ads }}\left(\mathrm{H}_{2}\right)\right.$, in $\left.\mathrm{kcal} / \mathrm{mol}\right)$ as a function of the surface atoms average $d$-band center $\left(\left\langle\varepsilon_{d}\right\rangle\right.$, in $\left.\mathrm{eV}\right)$, where $E_{\text {ads }}\left(\mathrm{H}_{2}\right)=$ $\frac{1}{m}\left[E\left(\mathrm{Ru}_{n} \mathrm{H}_{m}\right)-E\left(\mathrm{Ru}_{n}\right)-\frac{m}{2} E\left(\mathrm{H}_{2}\right)\right] . \tau$ is the $\mathrm{H}$ :surface $\mathrm{Ru}$ coverage value.

$E_{\text {ads }}\left(\mathrm{H}_{2}\right)$, on the surface of several $\mathrm{Ru}$ systems: the icosahedral $\mathrm{Ru}_{13}$, four $\mathrm{Ru}_{55}$ clusters (icosahedron, IC; cuboctahedron, CB; Marks decahedron-like, MD; spherical piece of an hcp cristal, HCP) and the flat $\mathrm{Ru}(0001)$ surface. $\left\langle\varepsilon_{d}\right\rangle$ is calculated as:

$$
\left\langle\varepsilon_{d}\right\rangle=\frac{\left(\sum_{\alpha \in \operatorname{surf}} \int_{E_{\min }}^{E_{F}} \epsilon n_{d}(\alpha, \epsilon) d \epsilon\right)}{\left(\sum_{\alpha \in \operatorname{surf}} \int_{E_{\min }}^{E_{F}} n_{d}(\alpha, \epsilon) d \epsilon\right)}
$$

Among the four $\mathrm{Ru}_{55}$ clusters, the HCP isomer is the most stable, whereas the IC isomer lies a few $\mathrm{kcal} / \mathrm{mol}$ above (see Table S1). According to electron-count rules, naked $\mathrm{Ru}_{n}$ clusters can accommodate a large number of surface ligands. The expected linear relationship between $\left\langle\varepsilon_{d}\right\rangle$ and $E_{\text {ads }}\left(\mathrm{H}_{2}\right)$ is observed for optimal coverage values, as a consequence of the balance between the stabilization of the metal core gained by the presence of surface species and the minimization of the steric hindrance between them. Several coverage values $(\tau)$ have been considered for each cluster, ranging approximately from $\tau=0.25$ to $\tau=2.0$, sometimes starting from different coordination of hydrides for the same $\tau$ value. The graph plot in Figure 1 confirms that the $d$-band center $\left\langle\varepsilon_{d}\right\rangle$ is a good indication of the adsorption strength at the surface of nanoclusters. But the point we specifically want to address in this paper is to go beyond the seminal formulation of the $d$-band center model, by predicting the activity and selectivity of local sites at the surface of metal NPs. It is now going to be examined by calculating the dissociative adsorption energy of $\mathrm{H}_{2}$ on several adsorption sites of the $\mathrm{Ru}_{55}(\mathrm{MD})$ cluster.

On-site dependence of the adsorption of $\mathrm{H}_{2}$ on $\mathrm{Ru}_{55}$. Beyond a certain size, it is experimentally known that RuNPs adopt a spherical hcp structure. It is the case of the $1 \mathrm{~nm} \mathrm{Ru}_{55}(\mathrm{MD}) \mathrm{NP}$ shown in Figure 1 , chosen owing to the topological diversity of its surface. It has been adapted from the $\mathrm{Cd}_{55}$ cluster previously studied by Doye. ${ }^{52}$ Although it has no overall order, strong structural preferences can be identified in this structure reminiscent of a Marks decahedron: ${ }^{53,54}$ one side can be seen as two $\mathrm{Ru}(0001)$ surfaces 


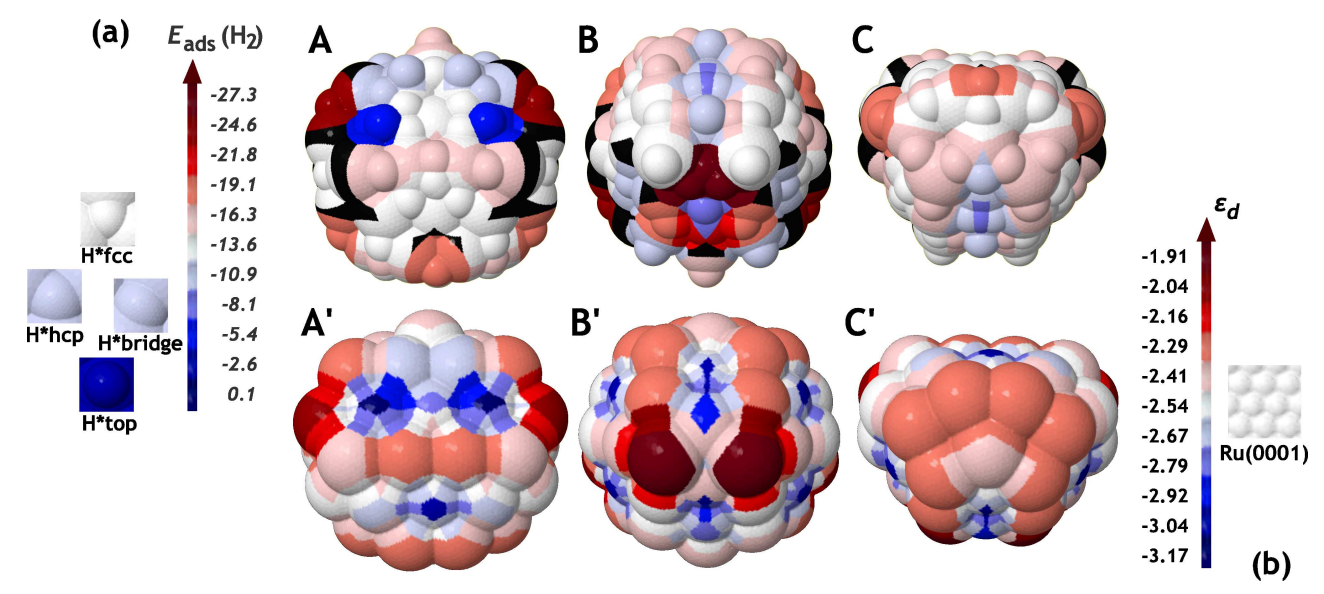

Figure 2: (a) Probing of the adsorption of $\mathrm{H}$ atoms at the surface of the $\mathrm{Ru}_{55}-\mathrm{MD}$ NP. (A-C) different points of view of the map summarizing the dissociative adsorption energy of $\mathrm{H}_{2}$. It is made by superposing $41 \mathrm{DFT}$ calculations performed on $\mathrm{Ru}_{55} \mathrm{H}$ nanoclusters, that is a total of 69 sites owing to the symmetry plane of the cluster. Each small CPK sphere is colored according to the energy scale (in $\mathrm{kcal} / \mathrm{mol}$ ) given on the right. Unprobed zones are shown in black. The corresponding mapping on the $\mathrm{Ru}(0001)$ surface is given in the insets (fcc: $-13.6 \mathrm{kcal} / \mathrm{mol}$, white; hcp and bridge: -11.9 and $-10.1 \mathrm{kcal} / \mathrm{mol}$, light blue; top: $-3.3 \mathrm{kcal} / \mathrm{mol}$, dark blue; values taken from ref 55). (b) $\bar{\varepsilon}_{d}\left(\mu_{k}\right)$ map of the $\mathrm{Ru}_{55}-\mathrm{MD}$ cluster and $\varepsilon_{d}$ energy scale. The orientations $\left(\mathbf{A}^{\prime}-\mathbf{C}^{\prime}\right)$ are given in the same order as in $(\mathbf{A}-\mathbf{C})$.

with a bending angle of $154^{\circ}$, whereas on the opposite side two $\mathrm{Ru}$ atoms are low-coordinate, owing to the presence of a subsurface defect. This structural feature is interesting inasmuch as it shows what the electronic fingerprint of such a defect in RuNPs would be. Forty-one adsorption sites were systematically considered. A color scale was adopted in maps A-C (Figure 2a). Such color maps give a straightforward indication of the adsorption strength of $\mathrm{H}$ atoms on all the sites probed; colored marbles correspond to $E_{\text {ads }}\left(\mathrm{H}_{2}\right)$, on the $\mathrm{Ru}(0001)$ plane (see ref 55 and references therein for a comparison with experimental values and other theoretical studies): white for $\mathrm{H}^{*} \mathrm{fcc}(-13.6 \mathrm{kcal} / \mathrm{mol})$, light blue for $\mathrm{H}^{*} \mathrm{hcp}(-11.0 \mathrm{kcal} / \mathrm{mol})$ and $\mathrm{H}^{*}$ bridge $(-10.1 \mathrm{kcal} / \mathrm{mol})$, and dark blue for $\mathrm{H}^{*}$ atop $(-3.3 \mathrm{kcal} / \mathrm{mol})$. Some interesting results can be extracted from these data: (i) $\mathrm{H}$ atoms adsorb on the atop, $\mu$ and $\mu_{3}$ sites of the $\mathrm{Ru}(0001)$-like facets with almost the same strength as calculated on $\mathrm{Ru}(0001)$ slabs (map A, Figure 2a); surface adsorption energies in the neighborhood of the subsurface defect are strong $(-27.3 \mathrm{kcal} / \mathrm{mol}$, map $\mathrm{B})$; two other sites located at sharp ridges also have the propensity to strongly adsorb $\mathrm{H}$ atoms $(-23.6 \mathrm{kcal} / \mathrm{mol}$, map $\mathbf{A})$; adsorption on edges is usually slightly larger than adsorption on flat surfaces (maps $\mathbf{A}$ and $\mathbf{C}$ ). It is intriguing that adsorption of $\mathrm{H}$ atoms on two kinds of sites is found to be twice as strong as on fcc sites of $\mathrm{Ru}(0001)$. It is clearly a matter of selectivity of given sites at the surface of this nanocluster, which cannot be explained by $\left\langle\varepsilon_{d}\right\rangle$.

Analysis of the on-site adsorption strength of hydrides. We shall now evaluate whether the $\varepsilon_{d}$ descriptor is able to account for these local adsorption strengths.

For this purpose, eq. 1 is now applied to each surface atom of a RuNP. In other words, this yields an effective $d$ energy level for each surface atom, $\bar{\varepsilon}_{d}(\alpha)$, which is expected to linearly correlate with adsorption energies of ligands.

Yet, by definition $\mu-\mathrm{H}$ and $\mu_{3}-\mathrm{H}$ atoms are shared by two or three Ru atoms. The possible correlation between $\bar{\varepsilon}_{d}(\alpha)$ and adsorption energies of $\mathrm{H}$ atoms on triangular facets or bridges cannot be evaluated by a standard plot of $E_{\text {ads }}\left(\mathrm{H}_{2}\right)$ versus $\bar{\varepsilon}_{d}(\alpha)$, which is an atomic descriptor related to atop adsorption only. A coordination counterpart of the atomic $d$-band center, $\bar{\varepsilon}_{d}\left(\mu_{k}\right)$, can however be calculated for each adsorption 
site by averaging the value of the Ru atoms possibly involved in such coordination. This is what is usually done to evaluate the $d$-band center value of special sites on surfaces, such as kinks or steps. Instead of plotting adsorption energies as a function of this coordination $d$-band center descriptor, we propose a visual indication given as a color map (its construction is explained in the Methods section). This color map gives an immediate view of the possible spots for strong (dark red), normal (white) and weak (dark blue) adsorption with respect to $\mathrm{Ru}(0001)$, the origin of the $\varepsilon_{d}$ energy scale being chosen as the value calculated for surface atoms of the [001] slab. The map obtained for $\mathrm{Ru}_{55}(\mathrm{MD})$ is given in Figure $2 \mathrm{~b}$, with the same points of view as the adsorption energies mapped in Figure 2a. There is a very satisfactory similarity between the $E_{\text {ads }}\left(\mathrm{H}_{2}\right)$ and $\bar{\varepsilon}_{d}\left(\mu_{k}\right)$ maps. The same dark blue sites associated to very weak adsorption strengths are found in both representations; the dark red spots are found in the same areas of the surface; adsorption energies close to the $-13.6 \mathrm{kcal} / \mathrm{mol}$ value found for the $\mathrm{Ru}(0001)$ surface are in line with $\bar{\varepsilon}_{d}\left(\mu_{k}\right)$ values close to the $d$-band center of the $\mathrm{Ru}(0001)$ plane, i.e. $-2.54 \mathrm{eV}$. Some discrepancies can be observed for certain coordination sites: adsorption energies calculated for edge sites (map C, Figure 2a) are between ca. $-19 \mathrm{kcal} / \mathrm{mol}$ and $-13.6 \mathrm{kcal} / \mathrm{mol}$, whereas the $C^{\prime}$ map suggests adsorption energies between -16 and $-19 \mathrm{kcal} / \mathrm{mol}$; the same comment applies to the ridge in the middle of maps $\mathbf{A}$ and $\mathbf{A}^{\prime}$. These moderate inconsistencies indicate that the accuracy of the $\bar{\varepsilon}_{d}\left(\mu_{k}\right)$ index in predicting adsorption energies of $\mathrm{H}$ atoms on the surface is around $\pm 3 \mathrm{kcal} / \mathrm{mol}$ for an energy range of $27 \mathrm{kcal} / \mathrm{mol}$ (i.e. $10 \%$ ). This value is low enough owing to the target of this work, i.e. finding an electronic descriptor that accounts for the adsorption energies calculated for the $\mathrm{Ru}_{55}(\mathrm{MD})$. It also confirms that the $\mathrm{Ru}(0001)$-like part of this small cluster is similar to an infinite $\mathrm{Ru}(0001)$ slab, both in terms of adsorption energy and electronic fingerprint. Other sites with adsorption energies higher or lower than the $-13.6 \mathrm{kcal} / \mathrm{mol}$ reference value are also well predicted by the $\bar{\varepsilon}_{d}\left(\mu_{k}\right)$ index, with the exception of the butterfly-like pattern that lies above the subsurface vacancy. It is clear from the $\mathbf{B}$ and $\mathbf{B}^{\prime}$ maps of Figures $2 \mathrm{a}$ and $2 \mathrm{~b}$ that to some extent the $\bar{\varepsilon}_{d}\left(\mu_{k}\right)$ index fails in accurately estimating the localization of the site that favors the strongest adsorption on $\mathrm{Ru}_{55}(\mathrm{MD})$. According to the map it should happen on the two apexes of the butterfly-like pattern lying above the subsurface vacancy (site b, Figure 3), whereas the strongest adsorption energy on atop sites is found on another Ru atom of the butterfly pattern (site $\left.\mathbf{b}^{\prime}\right)$. The local reorganization of the metal atoms of this pattern under $\mathrm{H}$ adsorption accounts for this difference. A careful similarity/structure analysis has shown that, upon adsorption, the atom moves away by $\sim 1.1 \AA$ from its initial position - a somewhat large value. The mobility of this atom upon adsorption thus involves electronic reorganization and the transfer of the enhanced adsorption property in the neighborhood of the site identified by our descriptor. This gives a special site status to this butterfly-like feature on the surface, both in terms of electronic structure and plasticity.

To conclude this part, the most promising success of the $\bar{\varepsilon}_{d}\left(\mu_{k}\right)$ index is its ability to quantitatively confirm that the highest adsorption energies calculated by this model cluster are related to the local availability of average $d$ orbitals. Now that this descriptor has shown its potential to deal with hydrides adsorbed on a $\lesssim 1 \mathrm{~nm}$ cluster, its properties must be further assessed: does it account for the adsorption of other model ligands on the surface of $\mathrm{Ru}_{n}$ nanoclusters?

Adsorption of $X$ and $\mathrm{L}$ ligands on $\mathrm{Ru}_{n}$ nanoclusters $(\boldsymbol{n}=55,147)$. Three other ligands were considered in addition to $\mathrm{H}$, namely the one-electron $\mathrm{CH}_{3}$ ligand, the two-electron $\mathrm{PH}_{3}$ ligand and $\mathrm{CO}$ ligands, the latter being a classical benchmark in surface- and nano-science. ${ }^{48}$ They were adsorbed on various atop, edge-bridging and face-capping coordination sites of low- and high-symmetry clusters, namely $\mathrm{Ru}_{55}(\mathrm{MD}), \mathrm{Ru}_{55}(\mathrm{HCP})$ and $\mathrm{Ru}_{55}(\mathrm{IC}), \mathrm{Ru}_{147}(\mathrm{IC})$. These extensive DFT calculations of adsorption energies are plotted in Figure 3 versus $\bar{\varepsilon}_{d}\left(\mu_{k}\right)$, in addition to selected cases of hydrogen adsorption. The first 
comment is that whatever the coordination site, the ligands adsorb in the order $\mathrm{CO}>\mathrm{PH}_{3}>\mathrm{H}>\mathrm{CH}_{3}$.

There is again a linear correlation between the adsorption energy of a ligand and $\bar{\varepsilon}_{d}\left(\mu_{k}\right)$. The main observation from Figure 3 is that the four slopes are very similar, with a significant dispersion. This confirms that there is an overall scaling behavior of adsorption energies that can be interpreted with the coordination $d$-band center. In other words, knowing both the adsorption energy of a ligand on a unique system ( $\mathrm{Ru}(0001)$, for instance) and $\bar{\varepsilon}_{d}\left(\mu_{k}\right)$ should yield a good estimate of the adsorption energy of the ligand on any site of a RuNP, provided that its atomic-projected DOS has been calculated. But can the scatter of adsorption energies be explained, or must this $\sim 6 \mathrm{kcal} / \mathrm{mol}$ deviation be considered as the intrinsic accuracy of such a simple monoelectronic descriptor? We are going to show that it does better than the apparent discrepancies observed in Figure 3. At first glance, the color map previously proposed is attractive because it is both simple and accurate in rationalizing the adsorption strength of $\mathrm{H}$ atoms on the surface of RuNPs. But coordination chemistry is more complicated than the simple interaction of the spherical $1 s \mathrm{AO}$ of an $\mathrm{H}$ atom with an effective $d$ orbital. This is at the basis of the versatility of the interaction between metal atoms and main group ligands and it is related to the local symmetry of the orbitals involved: the $d$ AOs of the metal and the $\sigma$ and $\pi$ active MOs of the ligands. This implies that the $d$-band center-based color map is a 0th-order tool that must be improved by subtle arguments in line with molecular orbital theory and local symmetry.

This is what we intend to show from now on by analyzing in detail the results obtained for the $\mathrm{CO}$ functional group. Firstly, the largest deviations are observed mainly for atop adsorption, i.e. on the $\mathrm{Ru}_{55}(\mathrm{IC})$ a, $\mathrm{Ru}_{55}(\mathrm{IC})$-c and $\mathrm{Ru}_{55}(\mathrm{MD})$-a sites. The adsorption energy of $\mathrm{CO}$ at an apex of $\mathrm{Ru}_{55}(\mathrm{IC})$ (a site) is significantly higher in energy than the values estimated from the $\bar{\varepsilon}_{d}\left(\mu_{k}\right)$ index, whereas it is the opposite for the a site in $\mathrm{Ru}_{55}(\mathrm{MD})$. But such discrepancies may not be surprising bearing in mind that, on the basis of ligand-field theory, an effective $d$ level is unable to account for the specific role of each $d$ AO in the bonding. This means that it is necessary to take into account the ligand-field splitting created on a given atom by all other atoms. Following symmetry arguments, we propose to interpret the apparent discrepancy observed for some atop coordination in terms of the availability of the metal $d$ orbital mainly involved in the atop bonding of $\sigma$-donor ligands, i.e. the $d_{z^{2}} \mathrm{AO}$. The farther the distance to the Fermi level, the weaker the vertical adsorption energy. Its effective energy, $\varepsilon_{d \sigma}$, is systematically indicated as an error bar in Figure 3. Note that it is meaningful for atop adsorption sites only (these properties are gathered in Table S4). The error bar is narrow in some cases, in relation with just a slight deviation from the linear scale. It is large in other cases, and nicely explains that calculated adsorption energies are much lower than expected on the apexes of the icosahedral clusters. The trend given by an error bar usually reduces the scatter. But the adsorption of CO on an edge of the $\mathrm{Ru}_{55}$ icosahedron (site $\mathbf{c}$ ) resists this simple rule of thumb. The $\left[\varepsilon-\varepsilon_{d \sigma}\right]$ energy range suggests that the adsorption energy of $\mathrm{CO}$ at site $\mathrm{c}$ should be lower than calculated. Actually, it turns out that the a and $\mathrm{c} \mathrm{Ru}_{55}(\mathrm{IC}) \mathrm{CO}$ structures are second-order saddle points which lead to $\mu$-CO and finally to $\mu_{3}-\mathrm{CO}$ (site $\mathrm{Ru}_{55}(\mathrm{IC})$-d in Figure 3). The preference of $\mathrm{CO}$ for a $\mu_{3}$ coordination site can be explained by analyzing in details the decomposition of the atomic $d$-projection of the DOS into its $\sigma\left(d_{z^{2}}\right), \pi\left(d_{x z}, d_{y z}\right)$ and $\delta\left(d_{x^{2}-y^{2}}, d_{x y}\right)$ components, which respectively account for terminal and tilted adsorption of $\sigma$-donor ligands and for in-plane interaction (Scheme 1). The values reported in Table S4 show that, on average, the $d_{\pi}$ effective $\mathrm{AOs}$ of edge atoms (structure $\mathrm{Ru}_{55}(\mathrm{IC})$-c) are higher in energy than the $d_{\sigma}$ effective $\mathrm{AO}$ $(-2.2 \mathrm{eV}$ vs. $-2.7 \mathrm{eV})$. The $d_{\pi}$ effective $\mathrm{AO}$ orientated perpendicularly to the edge actually lies even higher $(-1.9 \mathrm{eV})$. This suggests that a $\sigma$-coordinated ligand will rather be hooked by this $\mathrm{AO}$ and will consequently lie on the side of an edge. Owing to local symmetry, three edges will exert the same effect, resulting in the final $\mu_{3}$ preference of $\mathrm{CO}$ at the center of the triangular facets and in a strong adsorption energy ( -54.3 


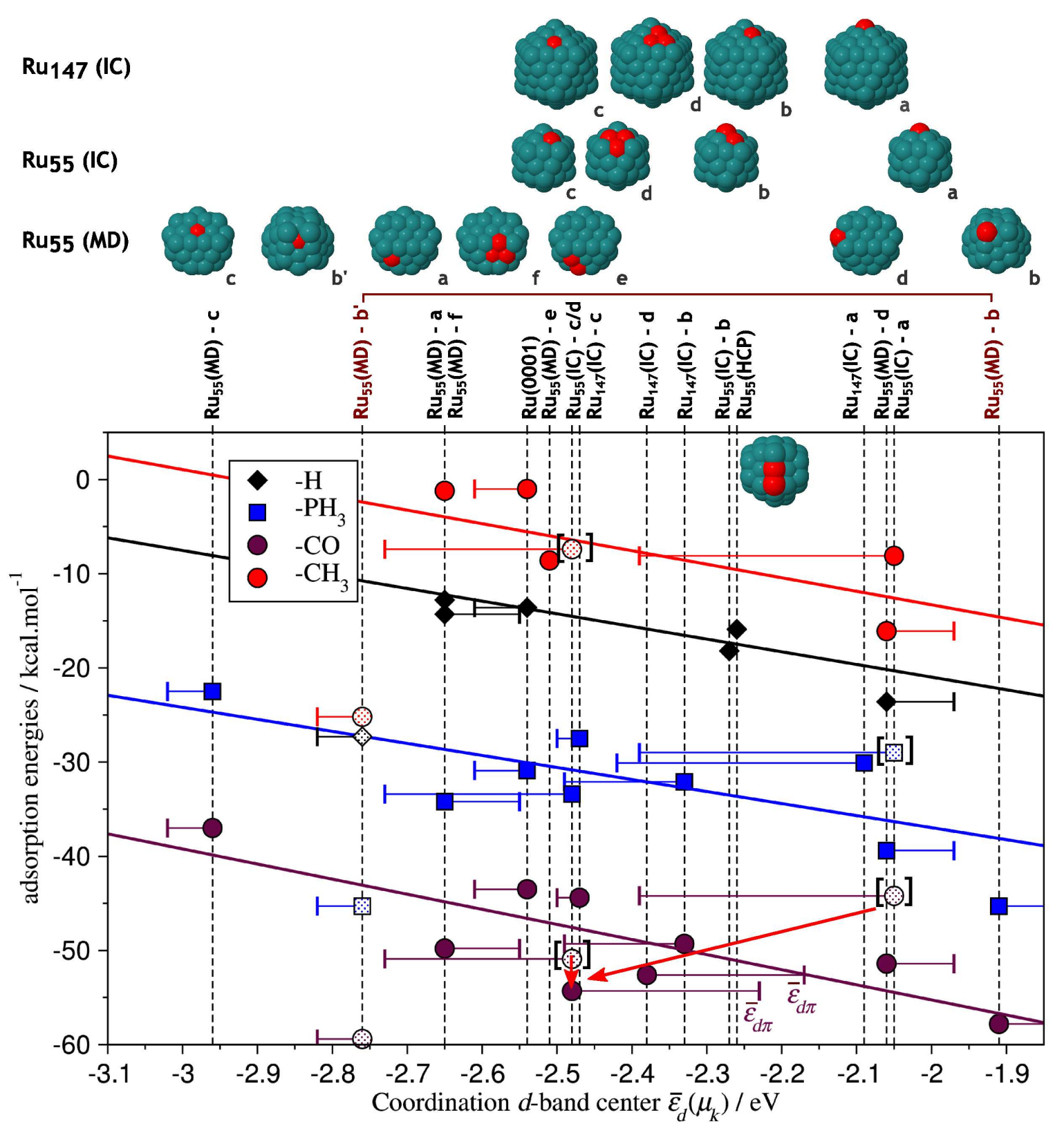

Figure 3: Adsorption energies (in $\mathrm{kcal} / \mathrm{mol}$ ) of $\mathrm{H}, \mathrm{CH}_{3}, \mathrm{PH}_{3}$ and $\mathrm{CO}$ as a function of the $d$-band center calculated for various coordination sites of $\mathrm{Ru}_{55}(\mathrm{MD}), \mathrm{Ru}_{55}$ (IC), $\mathrm{Ru}_{55}(\mathrm{HCP}), \mathrm{Ru}_{147}(\mathrm{IC})$ and on the $\mathrm{Ru}(0001)$ slab. The coordination sites are indicated in red on the $\mathrm{CPK}$ models. $\mathrm{CO}$ and $\mathrm{PH}_{3}$ are atop coordinated on $\mathrm{Ru}(0001)$, whereas $\mathrm{CH}_{3}$ and $\mathrm{H}$ are adsorbed on the fcc site. In an attempt to understand the scattering, the $d_{z^{2}}$-projected $d$-band center value $\left(\varepsilon_{d \sigma}\right)$ is indicated as an error bar for terminal ligands only. Another energy range based on the $d_{\pi}$-projected $\mathrm{d}$-band center (the definition of $\varepsilon_{d \pi}$ is given in the discussion section) is given for the $\mathbf{d}$ and $\mathbf{b}$ adsorption sites of and $\mathrm{Ru}_{147}(\mathrm{IC})$ respectively (see text for details). Second-order saddle points are also included on the figure, although they are not taken into account in the linear regression since these are metastable configurations (they are designated by square brackets, an arrow indicating which minima they connect for the $\mathrm{CO}$ case) 
$\mathrm{kcal} / \mathrm{mol})$. This is in line with the $\varepsilon_{d \pi}$ index of the Ru edge atoms it is connected to. Taking into account the relationship between each orbital-driven adsorption site and the $\varepsilon_{d}$ index each site is characterized by, significantly reduces the deviation with respect to the expected linear scaling, as indicated by the coefficient of determination, $R^{2}$. In the CO case, whereas it is found to be 0.62 by considering $\bar{\varepsilon}_{d}$, it increases to 0.83 by taking into account the appropriate index (see also Figure S2).

Let us now briefly focus our attention on the $\mathrm{L}=\mathrm{PH}_{3}$ and $\mathrm{L}=\mathrm{CH}_{3}$ cases. The deviations with respect to the interpolation lines are usually similar to those observed for the ruthenium carbonyl NPs: the $\mathrm{Ru}_{55}(\mathrm{IC}) \mathrm{L}-\mathrm{a}$ isomers lie significantly above the expected value; the $\mathrm{Ru}_{55}(\mathrm{MD}) \mathrm{PH}_{3}$-a isomer is found to be slightly more stable than the $\bar{\varepsilon}_{d}$ estimation; the plasticity of the $\mathrm{Ru}_{55}(\mathrm{MD})$-b' site involves a strong stabilization upon adsorption of $\mathrm{CH}_{3}$ and $\mathrm{PH}_{3}$; as suggested by the $\varepsilon_{d \sigma}$ index, the adsorption of $\mathrm{PH}_{3}$ on the $\mathrm{Ru}_{55}(\mathrm{MD})$-a site is enhanced compared to $\bar{\varepsilon}_{d}$. In short, there is an overall consistency between ligands that have a $\sigma$-donor character and which also interact with the metal sites either through $\pi$-backbonding or by hyperconjugation.

It is also interesting to note that the saddle-point pattern found for some atop adsorption is obtained in the case of a large conflict between the $d$ orbital availability of a site and the atop unavailability revealed by a low $\varepsilon_{d \sigma}$ value, i.e. in case of a large $\left[\varepsilon_{d}-\varepsilon_{d \sigma}\right]$ energy domain. No general rule can of course be drawn from such an observation, and a calculation of vibrational frequencies is the only appropriate way to identify saddle points. However, the computational cost of normal modes of vibration is prohibitive for the largest compounds and a guiding rule of thumb may be useful. All the cases investigated in this paper indicate that a strong contradiction between an energy-accessible $\varepsilon_{d}$ level and a deep $\varepsilon_{d \sigma}$ level may result in a lower atop adsorption strength than expected according to $\varepsilon_{d}$ or may reveal the transition state nature of the site.

Influence of the ligands on the electronic fingerprint of surface atoms. The influence of pre-adsorbed surface ligands on the adsorption strength of co-adsorbed species is also a key point. It deserves a dedicated study and we shall here only address the question of the modification of the coordination d-band center, $\bar{\varepsilon}_{d}\left(\mu_{k}\right)$, upon adsorption of $\mathrm{CO}$ ligands on the $\mathrm{Ru}_{55}(\mathrm{MD})$ model. In this preliminary study, we only considered the adsorption of $3 \mathrm{CO}$ ligands on the butterly-like site which lies above the subsurface vacancy (Figure $4 a)$. The average adsorption energy per $\mathrm{CO}$ group is $-48.4 \mathrm{kcal} / \mathrm{mol}$, i.e. still slightly larger than on the $\mathrm{Ru}(0001)$ surface $(-43.5 \mathrm{kcal} / \mathrm{mol})$ but significantly lower than the adsorption of a single CO on the optimal b' site $(-59.3 \mathrm{kcal} / \mathrm{mol})$. The deformation of the site which maximizes the adsorption strength for a single $\mathrm{CO}$ cannot simultaneously favor the co-adsorption of three $\mathrm{CO}$ groups to the same extent. It is interesting to note that the adsorption energy expected by plotting the $\varepsilon_{d}$ parameter averaged for these three atoms $(-2.25 \mathrm{eV})$ on the linear interpolation given in Figure 3 nicely fits the calculated value $(-50 \mathrm{kcal} / \mathrm{mol}$ vs. $-48.4 \mathrm{kcal} / \mathrm{mol})$. The $d$-band center map calculated for the $\mathrm{Ru}_{55}$ metallic core, plot on Figure $4 \mathrm{~b}$ can be compared with the B' map of Figure 2. With the exception of the three metal sites on which the CO groups are coordinated, the maps look very similar. We postulate from this single case that even in a small nanocluster, the species adsorbed only have a short-range impact on the electronic properties of the NPRu core and therefore on the adsorption energies of other species on the other sites.

\section{Discussion and refinement of the model}

On the necessity to improve the $\boldsymbol{d}$-band center. The $\bar{\varepsilon}_{d}\left(\mu_{k}\right)$ descriptor is an accurate index for explaining and estimating adsorption energies on all coordination sites available on the surface of ruthenium NPs. It was shown by the extensive study of $\mathrm{H}$ adsorption on several sites of a $\mathrm{Ru}_{55}(\mathrm{MD})$ model cluster and further confirmed by considering other $\mathrm{X}\left(\mathrm{CH}_{3}\right)$ and $\mathrm{L}\left(\mathrm{PH}_{3}\right.$ and $\left.\mathrm{CO}\right)$ ligands on $\mathrm{Ru}_{55}$ and $\mathrm{Ru}_{147}$ 

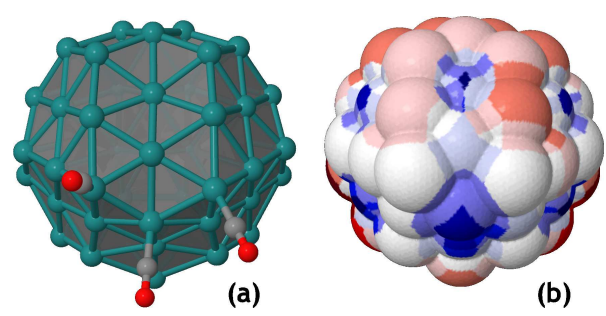

Figure 4: (a) geometry of a $\mathrm{Ru}_{55}-\mathrm{MD}(\mathrm{CO})_{3}$ cluster. The three carbon monoxide groups are adsorbed on the butterfly-like pattern; (b) $d$-band center map for the $\mathrm{Ru}_{55}-\mathrm{MD}$ metallic core (same orientationas $\mathrm{B}^{\prime}$ in Figure $2 \mathrm{~b}$; for the sake of clarity, the $\mathrm{CO}$ groups are hidden).

clusters. The associated trustworthiness range of this index is ca. $5 \mathrm{kcal} / \mathrm{mol}$. The strongest deviations were observed for saddle points, and on local plasticity of the surface. The so-called butterfly site turns out to be a prototype of the possible enhancement of the adsorption strength of a ligand upon surface deformation, in relation with the existence of a subsurface vacancy. In addition to this remarkable special site, the largest discrepancies, observed for atop adsorption, were mainly explained for $\mathrm{CO}$ by analyzing in detail the electronic structure of the metal surface and its compatibility with the electronic features of the ligands adsorbed. $\mathrm{CO}$ adsorption is ruled out by the interaction of the highly directional $\sigma \mathrm{HOMO}$ and of the $\pi^{*}$ MOs with the metal surface. But whereas the metal-to-ligand $\pi$ interaction is expected to be important for hollow site adsorption, it is weak for atop site adsorption. ${ }^{56}$ This suggests that the $\bar{\varepsilon}_{d}\left(\mu_{k}\right)$ index, averaged over the five $d$ components, may provide only a rough indication of the possible atop adsorption strength since the metal atomic orbital most involved in the bonding with $\mathrm{CO}$ is the $d_{z^{2}} \mathrm{AO}$. Our analysis has shown that our descriptor, although reasonably accurate, only provides a 0th order estimation of local adsorption energies. It has been improved by doing a projection along each $\sigma, \pi$ and $\delta$ component. This yields an indication of the propensity of a metal site to hook a ligand owing to the symmetry of the ligands' MOs primarily involved in bonding.

$\boldsymbol{d}$ AOs-projected maps. This is why we now propose to complete the $d$-band center map proposed in this study by $d_{\sigma}, d_{\pi}$ and $d_{\delta}$ maps, such as those plot in Figure 5a for $\mathrm{Ru}_{147}(\mathrm{IC})$ and $\mathrm{Ru}_{309}(\mathrm{IC})$. The high symmetry of these icosahedral RuNPs sheds light on the relationship between the local electronic fingerprint, adsorption properties and quantum-size effect (more usual energy-level diagrams are given in Figure S5). As already explained, such decomposition allows refinement of the conclusions arising from $\bar{\varepsilon}_{d}\left(\mu_{k}\right)$ and avoids spurious analysis. This is the case for the atop adsorption of ligands on the apexes and edges of these species. According to this descriptor it is expected to be stronger than the adsorption on $\mathrm{Ru}(0001)$, but the maps clearly show that the apparent availability of the effective $d$ level results from the high energy-lying $d_{\pi}$ and $d_{\delta}$ effective AOs. The $d_{\sigma}$ map is actually close to being white everywhere. This suggests an adsorption energy on apexes close to adsorption on $\mathrm{Ru}(0001)$. In contrast, the dark red zones in the $d_{\pi}$ and $d_{\delta}$ maps indicate that ligands with a highly directional $\sigma$ orbital should attack these sites sideways. According to local symmetry, the resulting adsorption could even be enhanced by a favorable $\pi$ interaction. It was observed for $\mathrm{Ru}_{55}(\mathrm{IC}), \mathrm{CO}$ adsorbing strongly at the center of triangular facets, in relation with a high $\varepsilon_{d \pi}$ index value (see Figure 3). Another important conclusion arising from these calculations of adsorption properties at the surface of the icosahedral RuNPs is that "edges" and "apexes" do not systematically mean "strong adsorption". It depends on the specific electronic hybridization involved by the local topology of the surface.

Quantum-size effect in RuNPs. It can be discussed by comparing the maps plotted for $\mathrm{Ru}_{147}(\mathrm{IC})$, $\mathrm{Ru}_{309}(\mathrm{IC})$ and $\mathrm{Ru}(0001)$ (Figures 5a and $5 \mathrm{~b}$ ). First, the apexes and edges of the two icosahedrons also have 
(a)

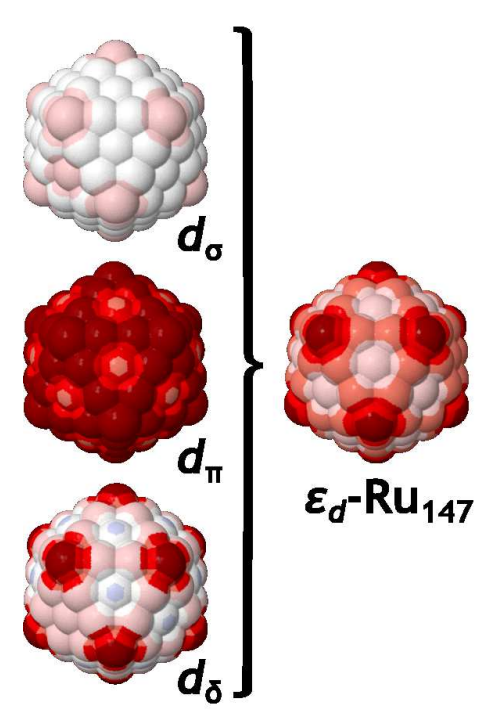

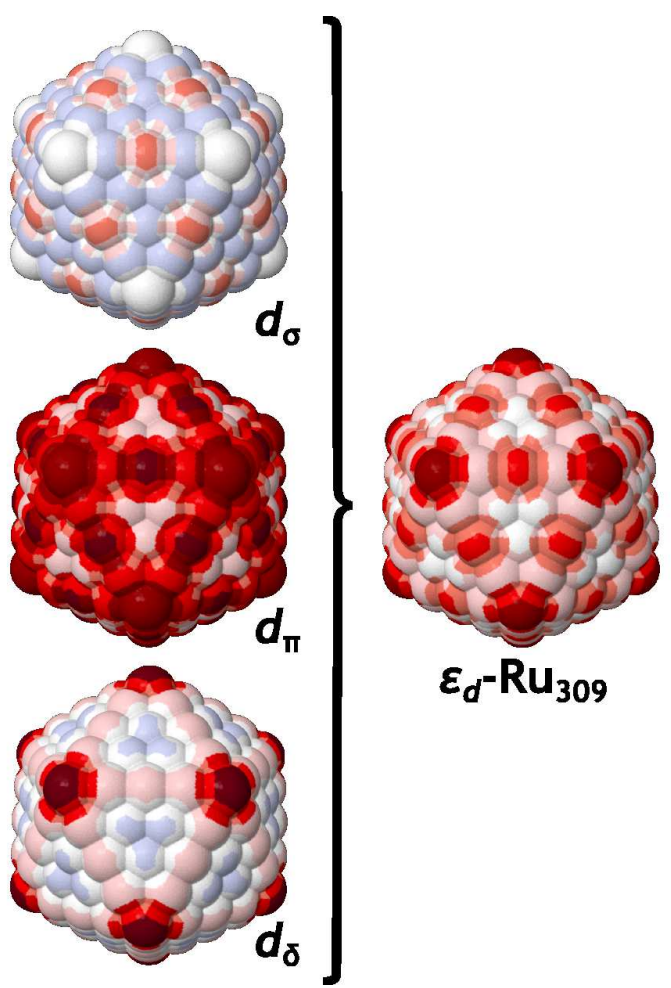

(b)

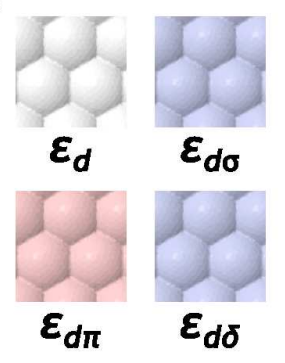

(c)

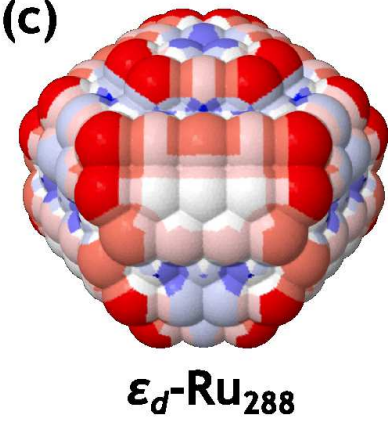

Figure 5: (a) $d$-band center coordination maps for $\mathrm{Ru}_{147}$ and $\mathrm{Ru}_{309}$ icosahedral NPs. The $d_{\pi^{-}}, d_{\delta^{-}}$and $d_{\sigma}$-projected maps are shown as well; (b) $\varepsilon_{d \sigma}(-2.61 \mathrm{eV}), \varepsilon_{d \pi}(-2.38 \mathrm{eV})$ and $\varepsilon_{d \delta}(-2.68 \mathrm{eV})$ maps calculated for the surface atoms of the Ru(0001) slab (see also Table S4); (c) $d$-band center map for an $\sim 1.8 \mathrm{~nm}$ $\mathrm{Ru}_{288}$ hcp NP. A Wulff construction gave the basic particle spherical shape. An edge row of atoms was removed between the (001) and (101) planes, giving rise to steps that exhibit $B_{5}$ sites. We followed the assumption formulated in ref 4 , later confirmed by DFT calculations, ${ }^{5}$ which showed that the $(001) /(101)$ edges lower their energy by a reconstruction in which the edge row of atoms is removed. According to $\varepsilon_{d}$, the most active sites for adsorption are the upper steps of the $B_{5}$ sites and the edges between the $\{110\}$ facets.

similar electronic fingerprints, with the exception of the atom that lies at the middle of the edge in $\mathrm{Ru}_{309}$. More interestingly, the atoms belonging to the triangular facets in this NP exhibit the same $d, d_{\sigma}, d_{\pi}$ and $d_{\delta}$ electronic features as the surface atoms of the $\mathrm{Ru}(0001)$ slab. As suggested by these indexes, although the triangular facets in $\mathrm{Ru}_{147}(\mathrm{IC})$ are far from being extended planes, adsorption of ligands on the central atom is expected to be only slightly stronger. Again, this means that it is the local topology of surfaces that rules the adsorption properties in large RuNPs; NPs that would mainly offer facets reminiscent of the $\mathrm{Ru}(0001)$ plane and a small number of special active sites should not exhibit a remarkable catalytic activity with respect to $\mathrm{Ru}(0001)$ heterogeneous catalysts.

Electronic fingerprint of the highly active B5 sites. The icosahedral structures are interesting because of their high symmetry which reveals global trends more clearly than clusters with an irregular surface. But it has been shown both experimentally ${ }^{57}$ and theoretically ${ }^{5}$ that colloidal RuNPs are spherical crystals that adopt the hcp structure of bulk ruthenium. Owing to this, our study would not be complete without an analysis of the electronic property of an hcp RuNP with realistic size. An important result in the context of adsorption properties on RuNPs is the presence of steps, the so-called $\mathrm{B}_{5}$ sites, ${ }^{4}$ that have been proven to be active with respect to the dissociation of the nitrogen molecule. ${ }^{5}$ Since hardly any $B_{5}$ sites will occur on small RuNPs, we considered a large spherical NP made of 288 atoms. Its $\bar{\varepsilon}_{d}\left(\mu_{k}\right)$ map is plotted in Figure $5 \mathrm{c}$ and the AO-projected indices are given in Table S4. It is remarkable that the upper steps of these special 
sites are characterized by a high $\varepsilon_{d}$ value $(-2.26 \mathrm{eV})$ - although they are not as active as the $\mathbf{b}$ and $\mathbf{b}$ ' special sites in $\mathrm{Ru}_{55}(\mathrm{DM})(-1.91 \mathrm{eV}$ for $\mathbf{b})$. But what is notable in the present case is that $d_{\sigma}, d_{\pi}$ and $d_{\delta}$ effective $\mathrm{AOs}$ are close in energy. This highlights the correspondence between a narrow bandwidth on such sites with a small degeneracy lift of the five effective $d$ AOs. They are all energetically available for subsequent reaction and this may give a versatile active property to these $B_{5}$ sites. Lastly, unlike the edges on the icosahedral NPs, those that also appear in red in Figure 5c (and in gray in Table S4) are also characterized by almost degenerate $d$ effective AOs. We can thus assume that these sites could also have a remarkable activity in catalytic processes involving RuNPs.

Practical interest of the scaling properties of the adsorption energies. The model has the power to predict adsorption energies of any ligand by the knowledge of its adsorption energy at a unique site. In order to illustrate this comment, let's consider the adsorption of the methyl tin group SnMe at the fcc site of the $\mathrm{Ru}(0001)$ surface. It is strongly bound by $-81 \mathrm{kcal} / \mathrm{mol}$, the largest value calculated in this work. As a consequence, such ligand, which could stem from the decomposition of $\mathrm{HSnMe}_{3}$, can efficiently poison the surface of Ru nanocatalysts. The scaling behavior observed in Figure 3 implies that its adsorption in the close neighborhood of the $\mathbf{b}$ and $\mathbf{b}$ ' site in $\mathrm{Ru}_{55}(\mathrm{MD})$ should be enhanced roughly by 10 to $15 \mathrm{kcal} / \mathrm{mol}$ (the slope is the same for all ligands, whereas the adsorption energy of $\mathrm{SnMe}$ on $\mathrm{Ru}(0001)$ yields the $y$ intercept). This is confirmed by DFT calculations $(-94.0 \mathrm{kcal} / \mathrm{mol})$. This further result confirms that the $\bar{\varepsilon}_{d}$ index provides good estimations for adsorption energies.

\section{Conclusion and outlook}

A new model accounting for the adsorption strength of ligands on the surface of Ru NPs has been developped in this paper. It is based on the $d$-band center model of Hammer and Nørskov, ${ }^{32}$ whose seminal formulation turns out to be a global property of metal surfaces or special sites, poorly able to accurately account for selective processes at the surface of transition metal NPs. Our model in contrast is defined as an on-site energy-weighted projection of the density of states on individual $d \mathrm{AOs}$. The resulting $\bar{\varepsilon}_{d}$ and $\varepsilon_{d_{m}}$ indexes for adsorption energy are conveniently depicted as color maps directly accounting for thermodynamics at the surface of the RuNPs. The most interesting sites according to the original averaged atomic $d$-band center $\bar{\varepsilon}_{d}$ can then be more deeply analyzed in terms of the $d$ AOs decomposition of this index and followed up by full DFT calculations. Such decomposition has a broader meaning and this is where the atomic $d$-band center proposed in this paper closely meets standard molecular orbital chemistry: it provides effective $d$ AO energy levels for each surface atom in its environment. In other words, it is the result of the ligand field generated by the other surface and core metal atoms, as well as by other surface species. It is a fascinating result since, to some extent, we can take advantage of the powerful rationalization offered by ligand-field theory for molecular systems in order to understand the chemistry of surface atoms in transition metal NPs. If M is a surface metal atom, its further adsorption ability is characterized by the energy splitting of its effective $d$ $A O s$; the adsorption of a ligand $L$ on this metal atom can then be understood in terms of a fragment orbital analysis of the reaction $M+L \rightarrow M-L$, and it may not be inappropriate to assume that a good knowledge of orbital interactions in chemistry ${ }^{49}$ could partially be transposed to understanding the local surface properties of metal NPs. According to our model, a RuNP must not be seen as a piece of metal with a delocalized electronic structure that rules adsorption on its surface: to some extent, it must be viewed as an assembly of mono- (atop), di- (edge-bridging), tri- (face-capping) or event tetra- $\left(\mu_{4}\right)$ nuclear complexes with effective electronic fingerprints resulting from interactions with other atoms. In other words, the adsorption strength 
on a site is directly related to the ligand field exerted by the other atoms and depends on the topology of this site. The local symmetry involves a specific energy splitting of the $d$ AOs, whereas the environment may modulate the amplitude of the splitting. On the basis of these DFT calculations, we have shown that, for Ru NPs, the ligand field is already as strong in small clusters as in model surfaces (see for example the planar facet of $\mathrm{Ru}_{55}(\mathrm{MD})$ vs. $\mathrm{Ru}(0001)$ and the $\mathrm{B}_{5}$ sites in $\mathrm{Ru}_{288}$ and in the $\mathrm{Ru}(10 \overline{15})$ slab). This is one of the very important outcomes of the present work.

Following all these assumptions and conclusions, we shall now propose to revisit the Sabatier principle. According to the qualitative concept of Paul Sabatier, catalytic properties will be hindered if the reactants adsorb too strongly, whereas no reaction will occur if the interaction is too weak. The Sabatier principle applied to the NP case is illustrated by the monoelectronic descriptors introduced in this paper and given as color maps: hot spots for adsorption are shown in red, blue identifies potentially weak interactions, whereas white shows where intermediate adsorption processes should occur. The arbitrariness of the color scale is obvious, but the white which is defined by the electronic feature of the $\mathrm{Ru}(0001)$ facet. Besides, the $\mathrm{B}_{5}$ sites appear in red, in agreement with a stronger adsorption strength of ligands on such special sites and with a well-known high activity. ${ }^{5}$ It may thus be assumed that the red color on these maps identifies ideal sites at the surface of Ru NPs. It may be the case, and $\bar{\varepsilon}_{d}$ can already be an appropriate descriptor for evaluating the catalytic performance of a site. But we postulate that the critical concept is that interactions between catalysts and reactants will be facilitated by the availability of the local electronic structure of the nanocatalyst. This property is quantitatively given by the $\varepsilon_{d \sigma}, \varepsilon_{d \pi}$ and $\varepsilon_{d \delta}$ descriptors, which allow to refine the conclusions based on the original on site $d$-band center, $\bar{\varepsilon}_{d}$ : a powerful catalytic site may be identified both by an appropriate $\bar{\varepsilon}_{d}$ value and by an appropriate ligand-field splitting. This was highlighted for the $\mathrm{B}_{5}$ sites, which are characterized both by a small degeneracy lift and a similar energetic availability of the five effective $d$ AOs of the upper step atom. Given the relationship between the shape and effective energy of these AOs (Scheme 1) and their overlap with the active MOs of an adsorbate (i.e. relevant to the catalytic process), we conjecture that a catalytic activity will be maximized by optimizing the energy of the effective $d$ AOs of the active sites. This can be achieved by modulating the ligand-field exerted on the metal atoms.

Another interesting conclusion of this work is the scaling properties of the adsorption energies, systematically shown whatever the ligand $\left(\mathrm{H}, \mathrm{CO}, \mathrm{PH}_{3}\right.$ and $\left.\mathrm{CH}_{3}\right)$. To the best of our knowledge, whereas they are now generally accepted for transition-metal surfaces, ${ }^{58}$ this is the first time that they have been found for individual adsorption sites at the surface of nanoparticles. The predictive character of this property has been checked for a tin ligand on one of the special sites considered in this paper.

Although our model is currently developed in the context of the PBE functional and PAW based periodic calculations, it is expected to exhibit the same consistency between adsorption energies and $d$-band center maps whatever the method for calculating the energy. It will be applied to other metals in order to quantify the variation in catalytic activities from one metal to another. However, several conclusions arising from this work are probably transferable to other medium-to-late transition metal NPs. A similar analysis for noble metal and magnetic NPs is in progress, the model being completed by taking into account the role of the $s$ density of states and $d$-band filling. The confirmation that activation energies of model reactions follow the trends given by the descriptors proposed in this paper is also investigated. The model is expected to contribute to bridging the gap between molecular inorganic chemistry and nanochemistry and it opens the route to the rational design of efficient transition metal nanocatalysts. 


\section{Methods}

DFT calculations. All computations were performed within the framework of the density functional theory (DFT) considering the spin unpolarized or polarized constraint, depending on the system under study. The exchange-correlation potential was approximated by the generalized gradient approach proposed by Perdew, Burke, and Ernzerhof (PBE) ${ }^{59}$. Calculation of energy parameters as well as geometry optimization were carried out using projector augmented waves (PAW) full-potential reconstruction ${ }^{60,61}$ implemented in the Vienna ab initio simulation package, VASP ${ }^{62,63}$. To minimize errors arising from the frozen core approximation, we used the PAW data sets treating the $4 p, 4 d$ and $5 s$ Ru states (14 valence electrons). As already proposed in a previous paper, ${ }^{55}$ we found that a kinetic energy cutoff of $280 \mathrm{eV}$ was sufficient to achieve a total energy convergence within several millielectronvolts for $\mathrm{H}$ adsorption. A cutoff of $500 \mathrm{eV}$ was used in the case of phosphine, methyl, tin and carbonyl compounds. For the geometry optimizations of clusters, a $\Gamma$-centered ${ }^{64}$ calculation was used, with a Gaussian smearing of $0.02 \mathrm{eV}$ width for the partial occupancies. Atoms were free to move until the residual forces on any direction were less than $0.02 \mathrm{eV} / \AA$. The supercell size is set to ensure a vacuum space of $c a$. $16 \AA$ between periodic images of $\mathrm{Ru}_{n}$ clusters $(25 \times 25 \times 26$ $\AA$ for $\mathrm{Ru}_{55}, 30 \times 30 \times 31 \AA$ for $\mathrm{Ru}_{147}$ and $45 \times 45 \times 46 \AA$ for $\mathrm{Ru}_{309}$ ). These parameters were tested and proven to be sufficient to accurately model the ruthenium clusters dressed by ligands for a reasonable computational cost. The DOS was determined by setting up the same parameters. The surfaces were modelled by a periodic six-layer metal slab, and the ligands were adsorbed on one side of the slab. The computational details are the same as those used in ref 55. Atomic and averaged $d$-band centers were calculated with our home-made tools4vasp suite of utilities. We used some test cases to check that we found the same $\left\langle\varepsilon_{d}\right\rangle$ values as the program developed by Henkelman's group. ${ }^{65}$ The harmonic vibrational modes were calculated for certain clusters in order to distinguish minima and saddle points by using the dynamical matrix code implemented in VASP as well as the VASPTST tools also developed by Henkelman's group. Adsorption energies were calculated according to the formula $E_{\text {ads }}(\mathrm{L})=\frac{1}{m}\left[E\left(\mathrm{Ru}_{n} \mathrm{~L}_{m}\right)-E\left(\mathrm{Ru}_{n}\right)-\frac{m}{k} E(\mathrm{~L})\right]$, where $\mathrm{L}=\mathrm{H}_{2}(k=2), \mathrm{CO}(k=1),\left(\mathrm{CH}_{3}\right)_{2}(k=2), \mathrm{PH}_{3}(k=1)$. In the case of $\mathrm{H}_{2}$ and $\mathrm{C}_{2} \mathrm{H}_{6}, E_{\text {ads }}$ is a dissociative adsorption energy: it provides the stabilization energy of $\mathrm{H}$ atoms or methyl groups, and includes the cost of a $\mathrm{H}-\mathrm{H}$ or $\mathrm{C}-\mathrm{C}$ bond breaking.

Construction of a $\boldsymbol{d}$-band center based coordination map. As explained earlier, we assessed the adsorption strength around each surface atom of ruthenium NPs by means of $\bar{\varepsilon}_{d}(\alpha)$, the energy-weighted DOS projected on all $d$ atomic orbitals of a surface atom $\alpha$. This atomic $d$-band center can be nicely depicted as a color map, which provides a first overview of the $d$-accessibility of an atomic site (see Figure 6, top representation). $\varepsilon_{d}$ is not expected to be accurate enough to account for slight differences in adsorption strength between two neighboring coordination sites. But the on-top representation does not explicitly show that simultaneous adsorption on two weakly-bonding (blue) and strongly-bonding (red) sites is expected to be moderate (white). As explained earlier, a coordination $d$-band center $\bar{\varepsilon}_{d}\left(\mu_{k}\right)$ color map was chosen as a better visual indicator of adsorption strength. $\bar{\varepsilon}_{d}$ for a coordination site $\mu_{k}$ is simply calculated as an average of the $\bar{\varepsilon}_{d}(\alpha)$ values of all surface metal atoms involved in such bonding (eq. 1).

The final map is obtained as a superposition of the $\bar{\varepsilon}_{d}$ values calculated for atop, edge-bridging, faceand square-capping sites (map in the upper part of Figure 6). The $d_{\sigma}, d_{\pi}$ and $d_{\delta}$ indices proposed in this work were obtained respectively by projecting the DOS on the $d_{z^{2}} \mathrm{AO}$ only, on the $d_{x z}$ and $d_{y z} \mathrm{AOs}$, and on the $d_{x^{2}-y^{2}}$ and $d_{x y}$ AOs: 


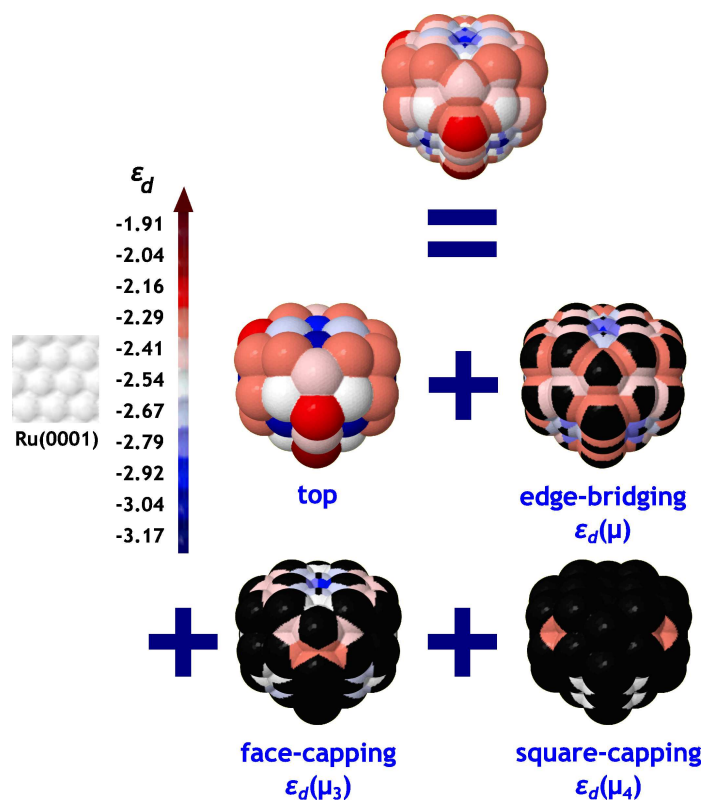

Figure 6: Construction of the $d$-band center-based coordination map as a superposition of the $d$-band center $\left(\varepsilon_{d}\right.$, in $\mathrm{eV}$ ) projected on all surface top, $\mu, \mu_{3}$ and $\mu_{4}$ coordination sites of the $\mathrm{Ru}_{55}$-hcp nanocluster. The correspondence between colors and energy scale (in $\mathrm{eV}$ ) is given on the left: the darker the red, the closer to the Fermi level; the darker the blue, the more stable the $d$-band center. The surface $\mathrm{Ru}(0001)$ average $d$-band center value $(-2.54 \mathrm{eV})$ was chosen as the origin of this scale whereas the top of the scale is set up to the highest $\varepsilon_{d}$ found in the calculated clusters and NPs, i.e. in the $\mathrm{Ru}_{55}(\mathrm{MD})$ nanocluster.

$$
\varepsilon_{d_{m}}(\alpha)=\frac{\left(\int_{E_{\min }}^{E_{F}} \epsilon n_{d_{m}}(\alpha, \epsilon) d \epsilon\right)}{\left(\int_{E_{\min }}^{E_{F}} n_{d_{m}}(\alpha, \epsilon) d \epsilon\right)}
$$

, where $m=z^{2}, x z, y z, x^{2}-y^{2}, x y$. Mind that the choice of coordinates is important since it is not unique. The $z$ axis was systematically set up as being orthogonal to the local average plane of the considered adsorption site, i.e. it is oriented along the on-top coordination site in the case of terminal coordination. The two $d_{\pi}$ and $d_{\delta}$ components may differ in some cases owing to local symmetry. For example, the in-plane $y$ axis was set up as collinear to edges, as shown in Scheme 1 . It involves that the $d_{\sigma}, d_{\pi}$ and $d_{\delta}$ effective energies plot as maps in Figure 5 or given in the SI (Table S4 and Figure S5) require to perform several DOS projections by setting up an appropriate orientation of the NPs as a function of each considered coordination site.

Acknowledgments. We thank the HPCs CALcul en Mldi-Pyrénées (CALMIP-Hyperion, grant P0611) and the Grand Equipement National de Calcul Intensif (GENCI-TGCC-Curie, grant 6211) for generous allocations of computer time. Financial support by the CNRS and the ANR (MOCA-NANO project, 2011INTB-1011-1) is gratefully acknowledged. This work has also benefited from stimulating discussions with Dr. B. Chaudret.

Supporting information available. Additional energy and structural information for $\mathrm{Ru}_{n}(\mathrm{~L})$ isomers; detailed decomposition of the atomic $d$-band center; cartesian coordinates of all compounds. This material is available free of charge via the Internet at http://pubs.acs.org. 


\section{References}

[1] Halperin, W. P. Rev. Mod. Phys. 1986, 58, 533-606.

[2] Taylor, H. S. Proc. R. Soc. London, Ser. A 1925, 108, 105-111.

[3] Hammer, B. Top. Catal. 2006, 37, 3-16.

[4] van Hardeveld, R.; van Montfoort, A. Surf. Sci. 1966, 4, 396-430.

[5] Honkala, K.; Hellman, A.; Remediakis, I. N.; Logadottir, A.; Carlsson, A.; Dahl, S.; Christensen, C. H.; Nørskov, J. K. Science 2005, 307, 555-558.

[6] Bond, G. C.; Coq, B.; Dutartre, R.; Ruiz, J. G.; Hooper, A. D.; Proietti, M. G.; Sierra, M. C. S.; Slaa, J. C. J. Catal. 1996, 161, 480-494.

[7] Six, C.; Beck, K.; Wegner, A.; Leitner, W. Organometallics 2000, 19, 4639-4642.

[8] Roucoux, A. In Topics in Organometallic Chemistry; Surface and Interfacial Organometallic Chemistry and Catalysis; C. Copéret, B. C., Ed.; Springer-Verlag: Heidelberg, 2005; Vol. 16; Chapter Stabilized Noble Metal Nanoparticles: An Unavoidable Family of Catalysts for Arene Derivative Hydrogenation, pp 261-279.

[9] Jansat, S.; Picurelli, D.; Pelzer, K.; Philippot, K.; Gómez, M.; Muller, G.; Lecante, P.; Chaudret, B. New J. Chem. 2006, 30, 115-122.

[10] Maitlis, P. M. J. Organomet. Chem. 2004, 689, 4366-4374.

[11] McQuire, M. W.; Rochester, C. H. J. Catal. 1995, 157, 396-402.

[12] Gerber, I. C.; Poteau, R. In Nanomaterials in catalysis; Serp, P., Philippot, K., Eds.; Wiley-VCH: Weinheim, 2013; Chapter Theoretical nanocatalysis: where are we now ?

[13] Niu, S.; Hall, M. B. Chem. Rev. 2000, 100, 353-406.

[14] Sautet, P.; Delbecq, F. Chem. Rev. 2010, 110, 1788-1806.

[15] Nørskov, J. K.; Abild-Pedersen, F.; Studt, F.; Bligaard, T. Proc. Natl. Acad. Sci. USA 2011, 108, 937-943.

[16] Shang, C.; Liu, Z.-P. J. Am. Chem. Soc. 2011, 133, 9938-9947.

[17] Chin, Y.-H. C.; Buda, C.; Neurock, M.; Iglesia, E. J. Catal. 2011, 283, 10-24.

[18] Liotard, D.; Penot, J.-P. In Numerical Methods in the Study of Critical Phenomena; Della Dora, J., Demongeot, J., Lacolle, B., Eds.; Springer: Berlin, Heidelberg, New-York, 1981; p 213, Proceedings of a colloquium, Carry-le-Rouet, France, June 2-4, 1980.

[19] Jónsson, H.; Mills, G.; Jacobsen, K. W. In Classical and Quantum Dynamics in Condensed Phase Simulations; Berne, B. J., Ciccotti, G., Coker, D. F., Eds.; World Scientific Publishing Co.: Singapore, 1998; Chapter Nudged Elastic Band Method for Finding Minimum Energy Paths of Transitions, pp 385-404.

[20] Sheppard, D.; Terrell, R.; Henkelman, G. J. Chem. Phys. 2008, 128, 134106-1-10.

[21] Remediakis, I.; Lopez, N.; Nørskov, J. Appl. Catal., A 2005, 291, 13-20.

[22] Lanzani, G.; Nasibulin, A. G.; Laasonen, K.; Kauppinen, E. I. Nano Res. 2009, 2, 660-670. 
[23] Jiang, T.; Mowbray, D. J.; Dobrin, S.; Falsig, H.; Hølbaek, B.; Bligaard, T.; Nørskov, J. K. J. Phys. Chem. C 2009, 113, 10548-10553.

[24] Viñes, F.; Lykhach, Y.; Staudt, T.; Lorenz, M. P. A.; Papp, C.; Steinrueck, H.-P.; Libuda, J.; Neyman, K. M.; Görling, A. Chem. Eur. J. 2010, 16, 6530-6539.

[25] Lanzani, G.; Laasonen, K. Int. J. Hydrogen Energy 2010, 35, 6571 - 6577.

[26] Pei, Y.; Shao, N.; Gao, Y.; Zeng, X. C. ACS Nano 2010, 4, 2009-2020.

[27] Roldan, A.; Manel Ricart, J.; Illas, F.; Pacchioni, G. Phys. Chem. Chem. Phys. 2010, 12, 10723-10729.

[28] Filot, I. A. W.; Shetty, S. G.; Hensen, E. J. M.; van Santen, R. A. J. Phys. Chem. C 2011, 115, 14204-14212.

[29] Kim, H. Y.; Lee, H. M.; Henkelman, G. J. Am. Chem. Soc. 2012, 134, 1560-1570.

[30] Fajin, J. L. C.; Bruix, A.; Cordeiro, M. N. D. S.; Gomes, J. R. B.; Illas, F. J. Chem. Phys. 2012, 137, 034701.

[31] Peterson, A. A.; Grabow, L. C.; Brennan, T. P.; Shong, B.; Ooi, C.; Wu, D. M.; Li, C. W.; Kushwaha, A.; Medford, A. J.; Mbuga, F.; Li, L.; Nørskov, J. K. Top. Catal. 2012, 55, 1276-1282.

[32] Hammer, B.; Nørskov, J. K. Surf. Sci. 1995, 343, 211-220.

[33] Hammer, B.; Nørskov, J. In Impact of Surface Science on Catalysis; Bruce C. Gates, H. K., Ed.; Adv. Catal.; Academic Press, 2000; Vol. 45; pp 71-129.

[34] Brönsted, J. N. Chem. Rev. 1928, 5, 231-338.

[35] Evans, M. G.; Polanyi, M. Trans. Faraday Soc. 1938, 34, 11-24.

[36] Sabatier, P. Ber. Dtsch. Chem. Ges. 1911, 44, 1984-2001.

[37] Toulhoat, H.; Raybaud, P. J. Catal. 2003, 216, 63-72.

[38] Swiegers, G. Mechanical Catalysis: Methods of Enzymatic, Homogeneous, and Heterogeneous Catalysis; John Wiley and Sons, Inc.: New-York, 2008.

[39] Tedsree, K.; Chan, C. W. A.; Jones, S.; Cuan, Q.; Li, W.-K.; Gong, X.-Q.; Tsang, S. C. E. Science 2011, 332, 224-228.

[40] Balandin, A. In Modern State of the Multiplet Theory of Heterogeneous Catalysis; D.D. Eley, H. P., Weisz, P. B., Eds.; Advances in Catalysis; Academic Press, 1969; Vol. 19; pp 1-210.

[41] Nørskov, J. K.; Bligaard, T.; Rossmeisl, J.; Christensen, C. H. Nat. Chem. 2009, 1, 37-46.

[42] van Santen, R. A.; Neurock, M.; Shetty, S. G. Chem. Rev. 2010, 110, 2005-2048.

[43] Jacobsen, C.; Dahl, S.; Clausen, B.; Bahn, S.; Logadottir, A.; Nørskov, J. J. Am. Chem. Soc. 2001, 123, 8404-8405.

[44] Nørskov, J. K.; Scheffler, M.; Toulhoat, H. MRS Bull. 2006, 31, 669-674.

[45] Anh, N. T. Frontier Orbitals: A Practical Manual; Wiley-VCH: Weinheim (Germany), 2007.

[46] Hammer, B.; Morikawa, Y.; Nørskov, J. K. Phys. Rev. Lett. 1996, 76, 2141-2144.

[47] Hoffmann, R. Solids and surfaces. A chemist's view of bonding in extended structures; Wiley-VCH: Weinheim, 1988.

[48] Stroppa, A.; Kresse, G. New J. Phys. 2008, 10, 063020. 
[49] Albright, T.; Burdett, J.; Whangbo, M.-H. Orbital interactions in chemistry; John Wiley and Sons, Inc.: NewYork, 1985.

[50] Fukui, K. Angew. Chem., Int. ed. Eng. 1982, 21, 801-809.

[51] Hoffmann, R. Angew. Chem., Int. ed. Eng. 1982, 21, 711-800.

[52] Doye, J. P. K. Phys. Rev. B 2003, 68, 195418.

[53] Marks, L. D. Rep. Prog. Phys. 1994, 57, 603-649.

[54] Yacamán, M. J.; Ascencio, J. A.; Liu, H. B.; Gardea-Torresdey, J. J. Vac. Sci. Technol., B 2001, 19, 1091-1103.

[55] del Rosal, I.; Truflandier, L.; Poteau, R.; Gerber, I. C. J. Phys. Chem. C 2011, 115, 2169-2178.

[56] van Santen, R. J. Mol. Struct. 1988, 173, 157-172.

[57] Pan, C.; Pelzer, K.; Philippot, K.; Chaudret, B.; Dassenoy, F.; Lecante, P.; Casanove, M.-J. J. Am. Chem. Soc. 2001, 123, 7584-7593.

[58] Abild-Pedersen, F.; Greeley, J.; Studt, F.; Rossmeisl, J.; Munter, T. R.; Moses, P. G.; Skúlason, E.; Bligaard, T.; Nørskov, J. K. Phys. Rev. Lett. 2007, 99, 016105.

[59] Perdew, J. P.; Burke, K.; Ernzerhof, M. Phys. Rev. Lett. 1996, 77, 3865-3868.

[60] Blöchl, P. Phys. Rev. B 1994, 50, 17953-17979.

[61] Kresse, G.; Joubert, D. Phys. Rev. B 1999, 59, 1758-1775.

[62] Kresse, G.; Fürthmuller, J. Phys. Rev. B 1996, 54, 11169-11186.

[63] Kresse, G.; Fürthmuller, J. Comput. Mater. Sci. 1996, 6, 15-50.

[64] Monkhorst, H. J.; Pack, J. D. Phys. Rev. B 1976, 13, 5188-5192.

[65] Froemming, N. S.; Henkelman, G. J. Chem. Phys. 2009, 131, 234103. 